THE magnetic and metabolic activational patterns of the brain during the perception, generation and silent articulation of words overlap to some extent, yet also measure concrete activational patterns. In the present study, auditory evoked magnetic fields (MEG) and changes in regional cerebral blood flow (PET) were examined in healthy subjects during a verb generation task. The aim of the study was to determine whether the advantages of both recording techniques can be combined so as to identify distributed sources of brain activity during particular tasks such as language processing. Given the currently observed disparity of the results from the two types of brain imaging we conclude that PET data will most likely not provide physiologically meaningful constraints for the distributed source analysis of MEG data, and may not necessarily validate results of distributed source analyses.

Key words: Magnetoencephalography (MEG); Eventrelated response; Positron emission tomography (PET); Activation studies; Language processing; Lexical stimuli

\section{Comparison of magnetic and metabolic brain activity during a verb generation task}

\author{
Carsten Eulitz, ${ }^{\text {CA }}$ Thomas Elbert, \\ Peter Bartenstein, ' Cornelius Weiller,' \\ Stefan P. Müller ${ }^{2}$ and Christo Pantev
}

Institute for Experimental Audiology, University of Münster, Kardinal-von-Galen Ring 10, D-48149 Münster; 'Department of Nuclear Medicine, University of München; ${ }^{2}$ Departments of Neurology and Nuclear Medicine, University of Essen, Germany

${ }^{\mathrm{CA}}$ Corresponding Author

\section{Introduction}

The investigation of humans is crucial for a neuroscientific understanding of speech and language processes and therefore requires non-invasive techniques. Positron emission tomography (PET) and magnetoencephalography (MEG) are two such methods which allow the mapping of functional brain activity. While PET provides unambiguous spatial information, its time resolution (minutes) is not sufficient to study the temporal interaction among speech-related centres such as Broca's and Wernicke's areas. MEG and EEG, on the other hand, provide an arbitrarily high temporal resolution, but seem to be hampered by the ambiguity of possible sources. While the position of a focal source can be detected within millimetres on the basis of MEG data, for analysis of distributed source configurations neither a single nor a multiple dipole model will provide adequate identification of the sources. A meaningful constraint for solving the inverse problem might be to restrict current dipoles to the grey matter of the brain with a perpendicular orientation to the cortical surface.' Reconstruction of the cortical surface based on magnetic resonance imaging (MRI) data has recently become available, but such modelling still awaits validation. Alternatively, meaningful constraints may be obtained from the spatial information of PET sources. The present investigation employed the latter technique: during verb generation, the possible sources of MEG activity were constrained to a spherical surface covering those centres which were active in the PET. This constraint was obtained for the group of investigated subjects, each of whom underwent both a PET and a MEG investigation and performed identical tasks during both measurements.

\section{Subjects and Methods}

After completing informed consent four subjects received the same stimulus material three times. The experimental design, including an example of the recorded MEG waveforms, is illustrated in Figure 1. A noun belonging to the lexical class of content words was presented acoustically every 5.7-6.3 $\mathrm{s}$ and the subject was asked to generate related verbs for each noun, e.g. if they heard the noun 'apple', one related verb might have been 'eat'. After a subject had generated a related verb and was ready to silently articulate the verb, he indicated the onset of the silent articulation by pressing a button. Prior to the experimental session subjects were trained to silently articulate the verbs without muscular activation.

During the PET session, two blocks of 30 words each were presented and compared with two blocks of a resting condition. During the MEG sessions four blocks of 30 words each were presented. In one MEG session the sensor array was positioned $1.5 \mathrm{~cm}$ over T3 (left hemisphere), in the other $1.5 \mathrm{~cm}$ over T4 (right

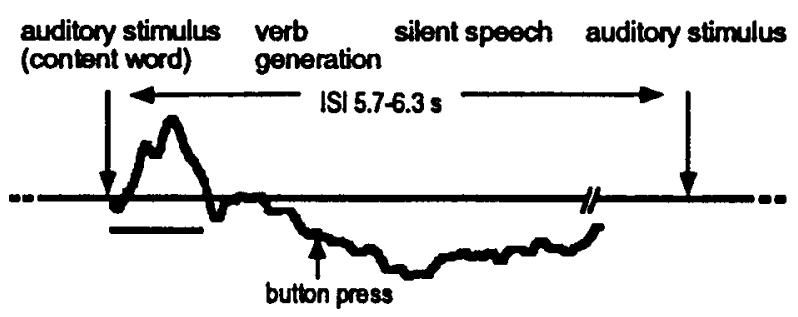

FIG. 1. Illustration of the experimental design and a basic magnetic waveform which appears in the course of one trial. 
hemisphere). The order of measurements was counterbalanced across subjects. For control purposes and to create an internal landmark for calculated dipole source locations, $1000 \mathrm{~Hz}$ tones were presented (ISI 1.8-2.2 s) 100 times per session in a separate experimental block after the main experiment. A 37-channel neuromagnetometer $\left(\right.$ Magnes $^{\circledR} \mathrm{BTi}$, consisting of firstorder axial gradiometers arranged in a circular array with diameter of $14.4 \mathrm{~cm}$ ) was used for MEG measurements (for details see Ref. 2). The EEG was monitored from 14 positions situated over the left hemispheric language areas; from its analogous electrode sites over the right hemisphere, and from $\mathrm{Fz}, \mathrm{Cz}, \mathrm{Pz}$. MEG and EEG were amplified from $\mathrm{DC}$ to $100 \mathrm{~Hz}$ and sampled at $297 \mathrm{~Hz}$. Regional cerebral blood flow (rCBF) was measured with PET (Siemens) using the $\mathrm{C}^{15} \mathrm{O}_{2}$ inhalation technique. The maximum of $\mathrm{rCBF}$ increase of each individual was determined by stereotactic normalization and statistic parametric mapping (for details see Ref. 3).

As well as standard analyses (for instance, latency and amplitude analysis, as well as localization of single moving equivalent current dipoles), MEG data were transformed to a set of distributed sources on a spherical surface to calculate minimum norm estimates. ${ }^{4}$ This approach was chosen because measured isofield contour maps showed (except for latency intervals around the N1m and the N4m waves) a pattern which could only be explained by the simultaneous activity of multiple or distributed sources. The location and diameter of the spherical surface was defined on the basis of the PET results such that the 'hot spots' identified in the PET coincided with the spherical surface. For data reduction, the calculated distributions of sources (grid for adjacent dipoles $=2 \mathrm{~mm}$ ) were submitted for an analysis of local maxima. For the final analyses, the local maxima fulfilling the following criteria were used as active sources. Relative criteria: for the actual time instance, every submitted local maximum has at least a dipole moment of $25 \%$ of the maximum dipole moment at this time (the percentage was chosen based on a simulation study). Absolute criteria: the dipole moment is larger than the median dipole moment in a fixed time interval of $0-1 s$ where the best signal to noise ratio occurred. This approach resulted in a consideration of the most powerful local maxima as active sources.

\section{Results}

In both hemispheres $\mathrm{rCBF}$ measured by PET was significantly enhanced above baseline. As illustrated in Figure 2, four 'hot spots' appeared in the left hemisphere and two 'hot spots' in the right hemisphere in all subjects. The maximum changes were located in the left superior temporal gyrus $(-54 /-40 / 8$ in Talairach coordinates; $z=6.0 ; z>2.58$ means $p<0.01$ ), which corresponds to the Wernicke area, and in the left

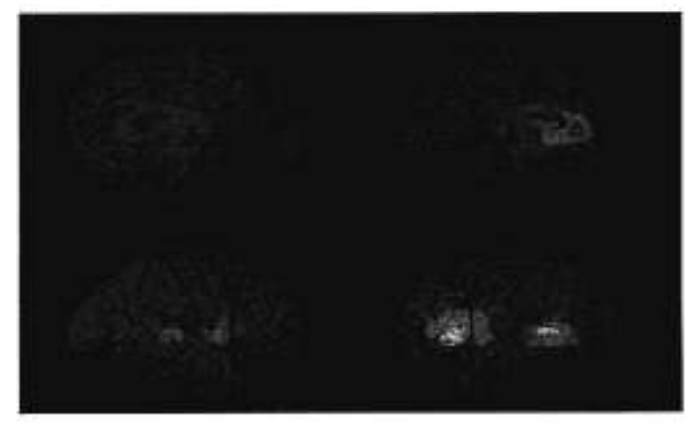

FIG. 2. Comparison of rCBF at rest and during the verb generation task The significance threshold for the statistic parametric images $(n=4)$ was set at 0.01 (Bonferroni corrected). Voxels representing a significant difference are displayed as sagittal projections on the lateral and medial surfaces of the left hemisphere (right side) and the right hemisphere (left side). The increasing significance level above 0.01 is indicated first by red and with an even greater increase in white.

inferior frontal gyrus $(-44 / 14 / 12 ; z=4.7)$, i.e. Broca's area, the left lateral prefrontal cortex $(-38 / 28 / 12$; $z=4.9)$ and the putamen $(-26 / 8 / 4 ; z=4.3)$. A less pronounced enhancement of the rCBF was obtained in the homologue regions to Wernicke's and Broca's area in the right hemisphere $(56 /-26 /-8 ; z=2.8$ and $44 / 8 / 8$; $z=2.9$ ).

The isofield contour maps of the MEG signals were explained in most instances by the activity of multiple sources. Figure 3 shows the projection of the submitted active sources to the spherical surface across time windows of $1 \mathrm{~s}$ for both hemispheres of all subjects during three different stages of the task. The first time window covered the period from 0 to $1 \mathrm{~s}$, i.e. word perception, the second time window was the $1 \mathrm{~s}$ interval just prior to the button press where subjects were mainly carrying out verb generation (median reaction time was calculated for each individual subject, and was $1.45 \mathrm{~s}$ for one subject and 2.0-2.8 s for the other subjects) and the third time window represents the 1 sinterval following the button press, i.e. the interval of the silent speech.

During word perception the primary and secondary auditory cortices in the left and the right hemisphere were activated. A part of the active sources were close to the single moving equivalent current dipole source of the tone evoked N1 $\mathrm{m}$ wave, a location which corresponds well to the auditory cortex. ${ }^{5}$ Additional sources anterior to the auditory cortex were activated in the left hemisphere of all subjects. In the right hemisphere the anterior sources were prominent in one subject only. Sources of magnetic brain activity posterior to the auditory cortex were seen for two subjects in the left hemisphere and for three subjects in the right hemisphere. Another activated area was found during word perception in the right hemispheric parietal cortex of three out of four subjects. This area remained active during the period of verb generation for two of these subjects and additionally in subject S4. In addition, the right hemispheric sources posterior to the auditory cortex were still active during verb generation in the 


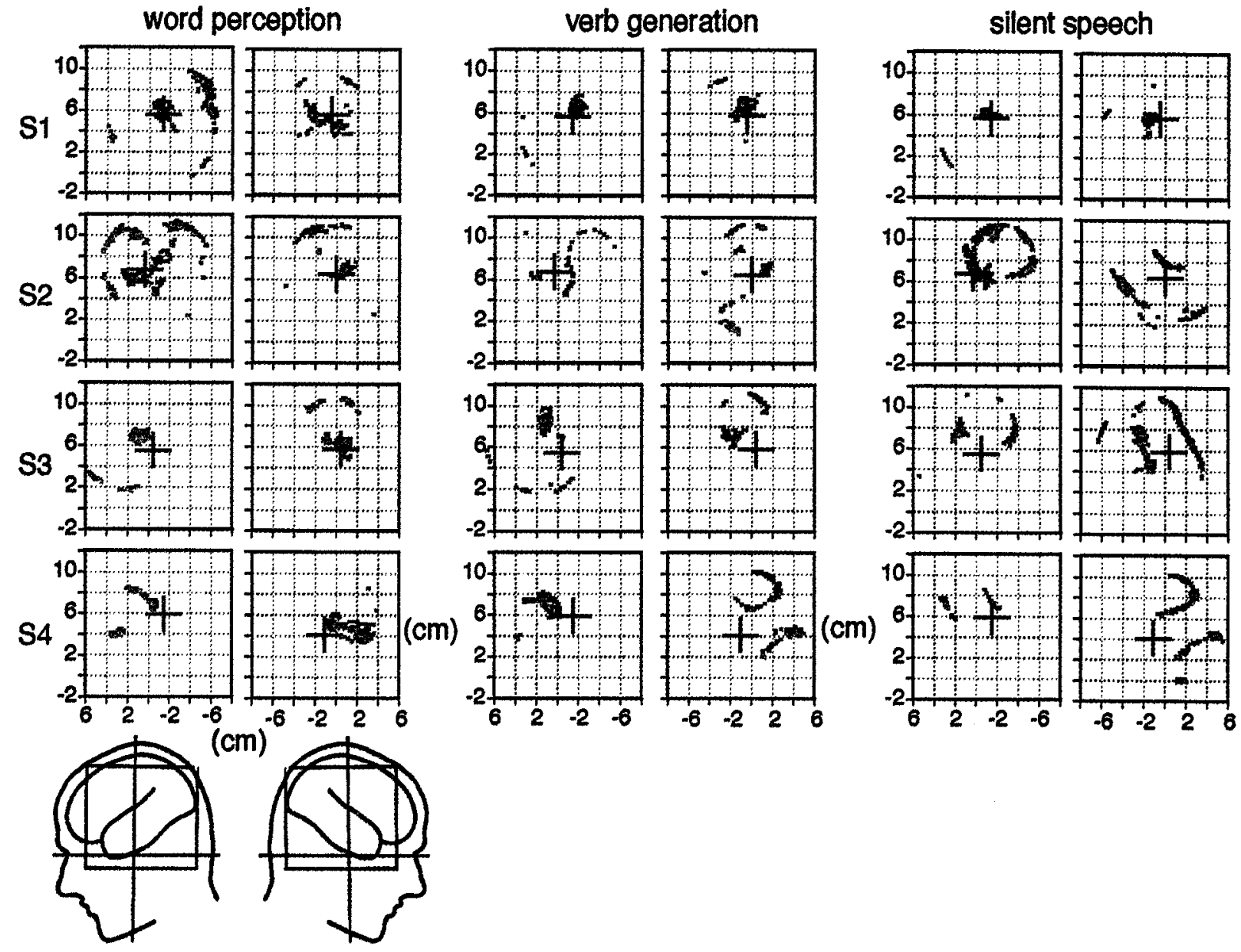

FIG. 3. Projection of the distributed magnetic source pattern on a spherical surface separately for all four subjects. Activation patterns were collapsed across $1 \mathrm{~s}$ intervals for the three stages of the verb generation task: the first pair of columns represents the word perception, the second pair of columns the verb generation and the third pair of columns corresponds to silent speech. As indicated by the scheme of the head, below, the left side of each column shows left hemispheric results and the right side the right hemispheric results. The total field of view is indicated by the rectangle, projected into the head scheme. + marks the projection of the single moving equivalent current dipole source of the N1m evoked by tones.

same three subjects. Furthermore, sources were seen in three subjects anterior to the auditory cortex, but not exactly at the same place as during the word perception. Other areas activated during the verb generation differed considerably across subjects. Note that in this period, except for subject S1, all active areas were separate from the auditory cortex. In the period of silent speech, the only repeatedly prominent activation pattern in more than two subjects was an active source anterior to the auditory cortex in the right hemisphere (in three out of four subjects). The other pattern again varied considerably across subjects during silent speech.

\section{Discussion and Conclusion}

The PET results revealed activation of Broca's and Wernicke's areas and their homologues in the right hemispheres. Further activation was observed in the left prefrontal cortex and in the left putamen during a verb generation task. This is in line with previous results reported in the literature. ${ }^{6,7}$ The results held true for the average across the whole verb generation task and for all subjects. Inspection of MEG data affirm activation of brain regions anterior and posterior to the auditory cortex which can be identified as Broca's and Wernicke's areas. MEG results further indicate sources in the primary and secondary auditory cortices, and, additionally, in parietal regions which were only partially in the field of view during the PET session. When comparing the gross MEG and PET activation pattern between the left and the right hemisphere (cf. Figs 2 and 3), the interhemispheric pattern differences for PET and MEG results were not very similar. Obviously, PET activation results are not linearly compatible with electromagnetic results. Variant physiological mechanisms underlying an $\mathrm{rCBF}$ increase and magnetic activity are likely sources for the quite deviant results. Therefore we conclude that PET data will most likely not provide physiologically meaning- 
ful constraints for the distributed source analysis of MEG data, and may not necessarily validate results of distributed source analyses. For an exact assessment of the congruence of activity centres calculated with both methods, technical problems must be solved and further data analysis must be carried out. Already now, however, several results are of interest, particularly the activation of brain regions anterior to the auditory cortex during word perception. Such results corresponds with the motor theory of speech perception ${ }^{8}$ and with the neurological theory of language, ${ }^{9}$ asserting that cell assemblies exist for the detection of linguistic signals which reach from Wernicke into Broca's area, as well as in the auditory cortex.

\section{References}

1. Lütkenhöner B. Möglichkeiten und Grenzen der neuromagnetischen Quellenanalyse. Münster, Hamburg: LIT, 1992: 96-104.

Pantev C Gallen C Hampson S et al. Am JEEG Technol 31 83-101 (1991).

Weiller C, Chollet F, Friston KJ et al. Ann Neurol 31, 463-472 (1992).

. Grunwald A, Lütkenhöner $B$, Menninghaus $E$ et al Analysis of auditory fields using a distributed source model. In: Deecke L, Baumgartner $\mathrm{C}$, Stroink $\mathrm{G}$ et eds. Recent Advances in Biomagnetism: Book of Abstracts. 136-137 (1993)

5. Pantev C, Hoke M, Lehnertz K et al. Electroencephalogr Clin Neurophysiol 75 , 173-184 (1990)

6. Wise $E$, Chollet F, Hadar $U$ et al. Brain 114, 1803-1817 (1991).

7. Petersen SE and Fiez JA. Annu Rev Neurosci 16, 509-530 (1993).

8. Liberman AM and Mattingly IG. Cognition 21, 1-35 (1985).

9. Braitenberg V and Pulvermüller F. Naturwissenschaften 79, 103-117 (1992)

ACKNOWLEDGEMENTS: Research was supported by the Deutsche Forschungsgemeinschaft (Ho 847/6) and grant EC-Biomed I CT 94/1261. 